\title{
tic\&société
}

Vol. 7, $\mathrm{N}^{\circ} 1$ | 1er semestre 2013

Formes et enjeux de la collaboration numérique

\section{Enseigner l'activité « écriture collaborative »}

Thibaud Hulin

\section{(2) OpenEdition}

\section{Journals}

Édition électronique

URL : http://journals.openedition.org/ticetsociete/1314

DOI : 10.4000/ticetsociete.1314

Éditeur

Association ARTIC

Référence électronique

Thibaud Hulin, «Enseigner l'activité « écriture collaborative » », tic\&société [En ligne], Vol. 7, № 1 | 1er semestre 2013, mis en ligne le 04 juin 2013, consulté le 19 avril 2019. URL : http://

journals.openedition.org/ticetsociete/1314; DOI : 10.4000/ticetsociete.1314 


\title{
Enseigner l'activité « écriture collaborative »
}

\author{
Thibaud HULIN \\ Université Montpellier 2 \\ Laboratoire LIRDEF \\ 2 place Marcel Godechot \\ BP 4152 \\ 34092 - Montpellier Cedex 5 \\ thibaud.hulin@univ-montp2.fr
}

Thibaud Hulin est maître de conférences en sciences de l'information et de la communication au Laboratoire Interdisciplinaire de Recherche en Didactique, Éducation et Formation (LIRDEF, Université de Montpellier 2) depuis 2011. Docteur en philosophie, ses recherches actuelles portent sur les dispositifs d'écriture numérique (collaborative, en réseau, multimédia...) et les outils innovants pour l'enseignement (TICE, web sémantique, vidéos, serious games). 
Thibaud HULIN

\begin{abstract}
Résumé
À l'heure où certaines politiques éducatives font de la "compétence collaborative " une clé du renouveau pédagogique, l'éducation à la collaboration numérique n'est pas prise en charge par l'école. Pour construire les conditions d'un enseignement durable, nous avons développé un modèle de formation centré sur l'autonomie de l'apprenant. Nous présentons les résultats d'une expérience menée avec des étudiants, notre conception du modèle pédagogique, puis la méthode de réalisation, d'observation et d'analyse de cette expérience. Nous avons analysé les discussions dans une messagerie instantanée, entre les étudiants et l'enseignant, et leurs productions. Tous les résultats montrent l'influence du dispositif de formation. Ainsi l'éducation à la collaboration numérique peut se construire à partir de l'apprentissage d'une triple capacité d'analyse : fonctionnelle, réflexive et critique ou comparative. Ce modèle pédagogique se situe à l'opposé d'une conception de la collaboration qui se réduirait au "bon usage " d'une interface. Notre modèle permet aux apprenants de développer une démarche d'autonomie, et d'acquérir un véritable savoirfaire technologique.
\end{abstract}

Mots-clés : écriture collaborative, éducation, clavardage, réflexivité, écriture numérique.

\begin{abstract}
Although certain education policies emphasize "the ability to collaborate" as a key element of educational reform, education for digital collaboration is poorly supported by public schools. To build the conditions of a sustainable education, we have developed a training model focused on learner autonomy. We present the results of an experiment conducted with students, our pedagogical model and its implementation, as well as our observation and analysis of this experiment. We analyzed discussions in instant messaging between students and teachers, and their productions. All results show the influence of our training. Thus, education and digital collaboration can be built from a triple analytic capacity: functional, reflexive and critical or comparative. This educational model refuses to reduce the concept of collaboration to being able to use an interface. It allows learners to develop their autonomy, and to acquire true technological know-how..
\end{abstract}

Keywords: collaborative writing, education, chat, reflexivity, digital writing. 
Enseigner l'activité « écriture collaborative »

\section{Resumen}

A pesar del hincapié que ciertas políticas de educación hacen en "la competencia de colaboración", como elemento clave de la reforma pedagógica, la educación en la colaboración digital, no se realiza en las escuelas. Para crear las condiciones de una educación sostenible, hemos desarrollado un modelo de formación centrado en la autonomía del alumno. En este artículo, se presenta nuestra concepción de modelo pedagógico y los resultados de un experimento realizado con los estudiantes, así como el método de realización, de observación y de análisis de dicho experimento. En una mensajería instantánea, entre estudiantes y profesores, se analizaron los debates ; los resultados muestran la influencia que ejerce el dispositivo de formación. De esta forma, la educación tendente a la colaboración digital puede ser construída a partir de tres capacidades de análisis: funcional, reflexiva y crítica o comparativa. Nuestro modelo educativo permite a los estudiantes desarrollar un aprendizaje autónomo, a la vez que adquirir un verdadero saber-hacer tecnológico.

Palabras clave: escritura colaborativa, educación, chat, reflexividad, escritura digital. 
Thibaud HULIN

La question de l'éducation à la collaboration numérique est-elle mineure par rapport à l'étude du pouvoir industriel qui la rend possible ? Nous pensons que ces deux types d'études ne s'opposent pas, mais relèvent d'un même effort de critique et d'information face aux industries et aux politiques de la collaboration actuelles. Ces dernières années, le développement des grandes industries du Web que sont Facebook ou Google ont suscité l'attention des sciences de l'information et de la communication qui ont pointé les risques de l'industrie du traçage (Arnaud et Merzeau, 2009). Ce développement va de pair avec le déploiement du Web dit « social » ou « 2.0 ») supposé " exploiter l'intelligence collective » (harnessing collective intelligence) (OReilly, 2007). Bien que cette exploitation industrielle des collaborations entraîne une idéologie enthousiaste autour des nouvelles technologies, celle-ci est susceptible de masquer les risques qu'elle comporte aux dépens des usagers. Par exemple, les utilisateurs les plus populaires des réseaux communautaires virtuels multiplient les messages dits " positifs " qui plaisent à leur communauté (Zhang, 2010), à l'opposé d'une démarche plus ouverte, de critique et de connaissance. Comment les systèmes éducatifs prennent-ils en charge cette situation ? Certaines politiques éducatives font de la " compétence collaborative " une clé du renouveau pédagogique (à partir du cadre de l'Union européenne (2006)), par exemple en France avec le rapport Fourgous (2012). Cependant, ces projets restent rares et surtout, nous ne savons pas comment prendre en charge de telles formations. Pour toutes ces raisons sociales, scientifiques, politiques, industrielles et éducatives, il devient urgent de construire les conditions d'une éducation à la collaboration numérique durable. Celle-ci doit trouver un équilibre entre l'enthousiasme de ceux qui font commerce de ces "dispositifs" collaboratifs, et l'attention sécuritaire de ceux qui en soulignent les risques. Pour répondre à ces exigences, nous devons abandonner le paradigme de la formation procédurale qui se contente de fournir des recettes pour utiliser telle version de telle interface vite dépassée. Car la collaboration relève de la communication, et implique des compétences complexes. Réduire la collaboration au «bon usage » d'une interface, ce serait nourrir une idéologie de la collaboration qui ne favorise pas l'autonomie et la créativité de l'apprenant mais tisse de nouveaux liens de dépendance.

Pour développer une approche durable et non procédurale de la formation numérique, il est donc nécessaire de construire des «dispositifs " de collaboration qui rendent l'utilisateur indépendant des interfaces rencontrées. Un dispositif est, selon Foucault et al. (1994, t. III, p. 299), un " ensemble résolument hétérogène comportant des discours, des institutions... du dit aussi bien que du non-dit ». inscrit à la fois dans une relation de pouvoir et de savoir. 
Enseigner l'activité « écriture collaborative »

Éduquer à la collaboration numérique revient donc à créer des dispositifs dans lesquels savoir et pouvoir interfèrent, où la technique n'est pas séparable de l'humain comme le soutient Simondon (1969). Ainsi compris, le projet de formation à la collaboration numérique soulève une triple difficulté : comment transmettre à l'usager un savoir qui lui donne le pouvoir de maîtriser l'ensemble des fonctionnalités d'un logiciel ? Comment le rendre vigilant face aux nouveaux enjeux soulevés par l'avènement du support numérique ? Comment le rendre conscient des dynamiques qui façonnent son activité cognitive et productive ? Face à ce questionnement triple (maîtrise, vigilance et conscience), nous proposons d'envisager le projet d'un enseignement collaboratif numérique à partir d'une triple compétence : l'apprenant peut comprendre le fonctionnement ou le potentiel des logiciels collaboratifs ; il peut réfléchir aux contraintes que font peser sur ses productions les interfaces utilisées ; il peut réfléchir à ses propres pratiques collaboratives à partir d'une démarche réflexive.

Pour répondre à ces exigences, nous avons conçu un dispositif de formation à l'écriture collaborative numérique. Nous avons expérimenté ce dispositif auprès d'étudiants. Nous avons ensuite évalué ce dispositif afin de mesurer son influence sur les pratiques de l'apprenant. Nous présenterons tout d'abord notre perspective théorique et ses implications pour la conception de notre dispositif de formation, puis nos méthodologies, enfin les résultats et leur analyse.

\section{De la théorie à la mise en pratique}

\subsection{L'analyse fonctionnelle et l'analyse réflexive}

Selon Vygotski (1985, p. 270), la zone proximale du développement (ZPD) de l'enfant indique l'écart entre son niveau de développement présent, qui est déterminé "à l'aide de problèmes résolus de manière autonome " et le niveau qu'il atteint " quand il résout des problèmes non plus tout seul mais en collaboration ". Ce concept fait partie de ce que l'on appelle la "théorie de l'activité ", laquelle se réfère à l'ensemble du mode d'apprentissage humain. Dans ce sillage, Engeström (1995) considère que le travail collaboratif est une forme de travail de groupe qui ne se réduit pas à la coordination (comme division du travail), mais inclut aussi des phases de coopération (de remise en question) et de co-construction (l'objet d'étude n'est pas stabilisé mais en construction au moment même du travail commun. Dans le cadre de cette théorie de l'activité dans lequel nous nous inscrivons, l'apprentissage est déterminé par l'histoire du sujet, mais aussi par ses interactions avec d'autres sujets, c'est-à-dire les collaborateurs, mais aussi l'enseignant dont la qualité de l'intervention est centrale. Ce positionnement dans les théories de l'activité suppose que l'activité collaborative, comme toutes les activités humaines, n'est 
Thibaud HULIN

pas réductible à une nomenclature ni à une grille standard qui inhiberait l'idée de co-construction. Penser l'activité collaborative revient donc à construire des grilles à partir de l'expérience réelle, et non a priori. C'est pourquoi nous écartons d'emblée les approches sur les standards de l'écriture collaborative (Lowry et al., 2004 ; Andriessen et al., 2011 ; Gunawardena et al., 2009 ; Joiner et Jones, 2003) qui semblent ignorer l'apport des théories de l'activité. Ces travaux tentent de construire des nomenclatures en amont de toute activité d'écriture réelle, au risque de gommer ces variations entre activité réelle et activité prescrite qui, pour nous, sont significatives et que nous cherchons justement à observer. Ce n'est pas le cas des travaux de Derobertmasure et Dehon (2009), qui proposent une synthèse récente de grilles d'analyse de l'activité professionnelle de l'enseignant du point de vue réflexif. Cependant, cette grille reste insuffisante lorsque l'on tente de la transposer aux jeunes adultes que nous avons observés, certaines catégories sont nettement surestimées (par exemple la catégorie « éthique » est très peu investie par les étudiants).

Sans a priori, nous proposons de repenser cette question fondamentale : comment l'enseignant peut-il intervenir pendant l'activité collaborative de l'apprenant ? L'enseignant va pouvoir fournir quelques connaissances utiles, comme par exemple apprendre à utiliser un logiciel. Aider l'apprenant à comprendre les potentialités d'un logiciel, c'est développer ses capacités d'analyse fonctionnelle, qui le rendent apte à repérer des fonctions, lesquelles associent des interactions à des actions de collaboration. Cependant, à ce stade, l'apprenant reste dépendant des procédures qui lui ont été fournies. S'il change de logiciel, les «recettes" qui lui ont été fournies ne sont plus opérantes. Pour s'adapter, il lui est alors nécessaire de réviser les routines pour saisir les principes de la collaboration numérique et les retrouver à partir de sa nouvelle interface. II doit alors réfléchir à son activité collaborative, à partir d'une "posture réflexive ». Cette hypothèse de l'apport réflexif pour l'apprentissage a été soutenue par de nombreux auteurs dans la lignée des travaux de Schön (1994). Tout en revendiquant cet héritage, Perrenoud (2001, p. 43) en vient à considérer que le « rapport réflexif à ce qu'on fait » est le pilier de l'autonomie. L'attitude réflexive est au cœur de la compétence dans la mesure où elle développe des capacités d' "auto-socio-construction ", "d'autorégulation et d'apprentissage à partir de l'expérience » (ibid).

Former à la collaboration numérique n'est donc pas seulement former à tel ou tel logiciel ou interface, mais aussi fournir des connaissances générales qui permettent à l'utilisateur de développer une pratique collaborative sans dépendre d'un outil particulier. Dans un environnement informatique, le but est de placer la compétence réflexive, cet « apprendre à apprendre » (Lloyd, 2003), 
Enseigner l'activité « écriture collaborative »

au centre de la culture numérique de l'apprenant, en l'aidant à analyser par luimême son activité. Nous faisons avec Flavell (1976, p. 232) l'hypothèse qu'il est central de développer la métacognition de l'apprenant, c'est-à-dire son activité cognitive en tant qu'elle est associée à des tâches « de contrôle et de régulation ", et à des stratégies et des réflexions portant sur cette activité. Aider l'apprenant à analyser son activité, c'est donc le confronter à des «traces » de son activité comme support de mémoire, hypomnemata (Foucault, 1994, t. II, p. 1237) et comme «écritures de soi »(Foucault, 1994, t. IV, p. 415 sq.)), dans le but de reconstruire l'activité de façon désintéressée. Le rôle de l'enseignant est alors d'accompagner l'apprenant dans le cadre de situations dites d' «autoconfrontations » (Clot et al., 2001 ; Theureau, 2010), où le sujet est confronté aux traces de son activité, pour lui permettre d'analyser et de prendre conscience des éléments critiques de son activité. Nous qualifierons alors l'ensemble des traces et productions de l'apprenant d' «écriture », voire d'écriture numérique s'il s'agit d'une activité médiée informatiquement, qu'il s'agisse de textes ou d'autres formes sémiotique du même genre (Christin, 2011).

\subsection{L'analyse critique ou comparative}

En associant les capacités d'analyse fonctionnelle et les capacités d'analyse réflexive de l'apprenant, nous développerons son autonomie. Pour autant, si l'activité de l'apprenant dépend de l'activité de ses collaborateurs, elle dépend aussi des outils qui sont à sa disposition. Plus particulièrement, les activités d'écriture collaborative dépendent des supports sur lesquels ils s'exercent. Goody (1979) considère que le support de l'écriture (tablette, papyrus, papier...) détermine non seulement l'activité d'écriture, mais aussi la pensée de celui qui écrit, sa « raison graphique ». À sa suite, Bachimont (2007) nous invite à réfléchir aux conséquences d'une " raison computationnelle », à partir de laquelle il propose une théorie du support selon laquelle le support d'écriture numérique contraint notre manière de penser. D'où la nécessité de former aussi l'apprenant à réfléchir au support numérique qui conditionne son écriture, ainsi qu'aux interfaces et aux dispositifs de collaboration dans une perspective critique. Bachimont propose par ailleurs de penser la formation au support numérique selon trois niveaux dits « du numérique ». En rejoignant cette approche dans son versant pédagogique, nous pouvons décrire l'activité collaborative numérique à partir de trois niveaux. Pour chacun de ces niveaux, nous envisagerons une activité critique et comparative dans notre formation à l'écriture collaborative :

1. le niveau fondamental est assimilable à ce qu'entend Manovich (2002, p. 53) par « représentation numérique » où tout est discret et calculable. À ce 
Thibaud HULIN

stade, les apprenants comparent les supports: ils comparent leur activité d'écriture collaborative sur papier et leur activité d'écriture collaborative sur un support numérique ;

2. le niveau applicatif désigne l'ensemble des contraintes d'écriture imposées par une interface, un logiciel ou un service Web : les apprenants comparent des logiciels collaboratifs et les contraintes qui en découlent ;

3. enfin, le niveau interprétatif renvoie à l'activité interprétative de l'utilisateur confronté aux formes sémiotiques qu'il perçoit : les apprenants comparent alors les processus et les productions des utilisateurs.

Ces trois niveaux vont nous permettre d'organiser notre dispositif pédagogique selon trois types de connaissances qui guident l'activité du sujet :

- des notions internes aux interfaces abordées, les fonctionnalités de l'application, qui requièrent des capacités d'analyse objective (analyse fonctionnelle) ;

- des prises de recul réflexives qui concernent la connaissance que le sujet a de sa propre activité (analyse réflexive) ;

- des notions ou principes de haut niveau quelles que soient les interfaces auxquelles il a affaire, et qui dépendent du support numérique (analyse critique).

\section{Méthodologies}

Nous décrivons dans cette section la manière dont nous avons conçu notre dispositif pédagogique et la méthode que nous avons suivie pour l'observation de sa mise en œuvre.

\subsection{Description du dispositif pédagogique}

\subsubsection{Contexte}

La formation que nous présentons dans ce document s'inscrit dans le cadre d'une série de formations financées par le projet régional français PRECIP1, au collège et à l'université, notamment en proposant des activités d'écriture collaborative, hypertextuelle (dans un blog), interactive (sur une carte

PRECIP : PRatiques d'ÉCriture Interactive en Picardie : http://www.precip.fr 
Enseigner l'activité « écriture collaborative »

géographique) et sur un réseau dit social. Chaque formation se centre autour d'une activité. Afin de ne pas alourdir notre propos, nous traiterons dans cet article de l'activité d'écriture collaborative réalisée à l'université.

\subsubsection{Modèle suivi}

Nous avons unifié ces formations à partir d'un modèle qui suit rigoureusement les objectifs pédagogiques décrit ci-dessus (développer des compétences fonctionnelles, réflexives et critiques), et les prescriptions relevées dans le cadre théorique décrit ci-dessus. Dans certains cas cependant, nous avons enrichi notre approche par notre expérience pédagogique. Par exemple, tandis que la théorie des trois niveaux nous permet de concevoir trois phases d'apports réflexifs, deux autres phases (découverte et littérature numérique) ont été décidées à la suite de certaines expériences qui ont mis en évidence la nécessité de présenter le logiciel et de fournir des exemples originaux. Enfin, ce modèle doit beaucoup à l'approche pédagogique de Bouchardon et al. (2011) .

Chaque formation se divise donc en cinq sessions dispensées de façon continue :

1. Phase de découverte d'un logiciel : l'apprenant découvre le fonctionnement minimal d'un logiciel libre qui lui permettra de réaliser son activité d'écriture.

2. Apport réflexif au niveau applicatif : l'apprenant compare l'application qu'il utilise à une autre qu'il connaît, par exemple OpenOfffice Writer et Facebook. II réfléchit alors aux principes fonctionnels qui structurent son écriture : dans quelle mesure le logiciel invite-t-il les participants à écrire d'une certaine manière ? L'idée est que le dispositif n'est pas neutre et influence le processus de production ou de communication : il s'agit de découvrir quelques principes ordonnateurs. L'apprenant doit parvenir à analyser les fonctionnalités du logiciel et, de façon plus générale, le fonctionnement d'un échange collaboratif médié par ces interfaces (analyse fonctionnelle).

3. Apport réflexif au niveau théorique : l'apprenant compare les supports de communication : comment aurait-il fait avec un papier et un crayon, avec un téléphone ? II s'agit de comprendre cette fois comment le support conditionne l'écriture collaborative. Comparer les supports, les applications, les processus et les productions permet à l'apprenant de développer un point de vue critique et non pas purement descriptif (analyse critique ou comparative).

4. Illustration à l'aide d'exemples tirés de la littérature numérique. Bootz (2006) définit la "littérature numérique " ainsi : " toute forme narrative ou poétique qui utilise le dispositif informatique comme médium et met en œuvre 
Thibaud HULIN

une ou plusieurs propriétés spécifiques à ce médium ». L'apprenant tentera donc d'étudier en quoi un dispositif artistique numérique montre et tente de dépasser les contraintes vues précédemment, sur les plans applicatifs et théoriques. Après que les apprenants ont découvert et manipulé ces œuvres, l'enseignant discute avec eux à propos de leur impression ; il interprète ce ressenti à l'aide des concepts abordés aux niveaux applicatif et théorique.

5. Apport réflexif au niveau interprétatif : l'apprenant compare différentes pratiques d'écriture relatives à un même support, par exemple des blogs relevant d'un même genre, relatifs à un centre d'intérêt commun. L'apprenant tente d'analyser son activité lorsque nous lui restituons ses traces numériques à partir d'une démarche réflexive (analyse réflexive) ;

Chacune des sessions ci-dessus sont organisées en trois temps :

- un cours qui se présente sous la forme d'une démonstration ou d'un exposé réalisé par l'enseignant qui présente des dispositifs ou des productions, et expose les principes qui les organisent ;

- une mise en pratique du cours, faite par les apprenants : la continuation d'un exercice dont la consigne de production collaborative a été donnée au début de la formation. Ainsi, chaque apport théorique est suivi par un exercice pratique qui permet à l'apprenant de mettre en œuvre les principes abordés, ou bien d'imaginer d'autres voies de créativité.

- une discussion entre les apprenants et l'enseignant, qui confronte les utilisateurs à leurs traces d'activité, récolte leurs impressions et interprète leur activité à l'aide des concepts enseignés ou en référence aux exemples abordés.

\subsubsection{Tâches spécifiques à l'activité étudiée}

Le thème traité par les apprenants était les soulèvements du "Printemps arabe ". La tâche des apprenants que nous présentons dans ce document a consisté à effectuer une recherche d'information sur ce thème choisi avec eux, afin de réaliser une synthèse de leurs recherches par écrit, dans le cadre d'un module de "culture numérique et informationnelle ». Toutes les sessions de la formation ont été suivies durant la même après-midi pendant trois heures, en présentiel avec les étudiants.

Dans le cas de notre expérience d'écriture collaborative synchrone, les temps de pratique sont au nombre de quatre et non de cinq : il n'y a pas eu de pratique après le niveau théorique, afin d'alléger la séance, et aussi parce que 
Enseigner l'activité « écriture collaborative »

la présentation de la littérature numérique a pour but d'illustrer ce haut niveau d'abstraction.

Le logiciel qui a permis l'animation du cours que nous traitons ici est le service en ligne Etherpad ${ }^{2}$ pour l'écriture collaborative synchrone.

Pour la session littérature numérique, nous utilisons les œuvres de J.-P. Balpe (Rien n'est sans dire - Mail roman ${ }^{3}$ ) ainsi que Venus Poetry ${ }^{4}$, des œuvres célèbres en ligne qui ont été choisies car il s'agit soit d'une production collaborative, soit d'une invitation à collaborer. Le « mail-roman » permet à un « méta-auteur » de mettre bout à bout les propositions de textes que les participants lui fournissent. Pour comprendre le processus collaboratif, l'enseignant demande aux étudiants de modifier un article de l'encyclopédie Wikipédia, ainsi que de modifier ou de créer un poème illustrant une partie du tableau de la Vénus de Milo de Boticelli, repris dans le projet du collectif Venus Poetry.

\subsection{Méthode d'observation}

\subsubsection{Participants}

Dans cette formation, nous avons observé huit étudiants en formation à l'université de Paris VIII, âgés de 20 ou 21 ans. Ils suivent une formation en sciences de l'information et de la communication, en troisième année de licence, au sein de laquelle ils sélectionnent certaines options de cours. Ainsi, tous les étudiants ne travaillaient pas ensemble avant la formation.

Pendant les discussions collectives organisées dans le troisième temps de chacune des sessions de la formation, l'enseignant guide les apprenants afin de les aider à décrire leur activité d'apprentissage. Pour cela, nous nous appuyons sur la méthode des focus groups (Krueger et Casey, 2008). Cette méthode permet de dynamiser des groupes restreints relativement homogènes, partageant un langage commun, en vue d'un objectif déterminé : décrire " objectivement " l'activité d'apprentissage, et non la justifier, afin de favoriser certaines prises de conscience. Un guide d'animation a été fourni aux trois observateurs qui accompagnaient l'enseignant, afin de respecter certaines règles comme éviter les questions en « pourquoi » qui poussent l'apprenant à se justifier plutôt qu'à décrire l'activité. Enfin, trois observateurs accompagnent l'enseignant qui lui est en situation d'observation participative, afin d'effectuer des analyses distanciées.

2 Etherpad : éditeur de texte collaboratif en temps réel, http://www.etherpad.org

$3 \mathrm{http} / / /$ www.ciren.org/ciren/productions/mail-roman/

4 http://www.venuspoetry.com 
Thibaud HULIN

\subsubsection{Instrument pour l'analyse des données}

Pour l'analyse des discours, les premières hypothèses ont été fournies en utilisant le logiciel de Ratinaud et Dejean (2009), Iramuteq ${ }^{5}$, qui est un logiciel libre semblable à Alceste ${ }^{6}$, basé sur le logiciel de statistiques $R^{7}$. Ce logiciel permet, après lemmatisation et suppression du vocabulaire courant, de repérer les tendances lexicales des locuteurs et leurs "mondes lexicaux " dans lesquels se construisent les représentations des locuteurs. Nous avons ensuite réalisé une analyse des discours par des aller-retours entre les termes proposés par le logiciel et leurs implémentations dans le contexte discursif, notamment en faisant appel aux enregistrements.

\subsubsection{Collecte des données}

A l'issue des formations, l'analyste dispose d'un ensemble de traces d'activité hétérogènes : enregistrements audio, prises de notes, genèse du texte enregistrée par le logiciel (fonctionnalité Time Slider d'Etherpad), traces mnésiques et témoignages des apprenants confrontés à leur activité. Les discours, depuis l'énonciation jusqu'à l'analyse finale, subissent donc une série de transformations :

- 1. enregistrement des discours ;

- 2. travail de la bande son ;

- 3. écoute et écriture du discours oral ;

- 4. nettoyage du code, correction, normalisation en fonction des règles de codage choisies ;

- 5. transformation du code obtenu vers un code préparé pour Iramuteq ;

- 6. affichage des classes de vocabulaire et analyse ;

- 7. éventuellement, durant le cycle, retour sur une des étapes précédentes pour préciser les réglages.

- Pour terminer, nous effectuons quelques allers-retours entre ces différents matériaux en observant le détail de l'activité dans une perspective ethno-méthodologique.

5 Iramuteq: "Interface de $R$ pour les Analyses Multidimensionnelles de Textes et de Questionnaires », http://repere.no-ip.org/logiciel/iramuteq

6 Alceste : «Logiciel d'Analyse de Données Textuelles » issu du C.N.R.S. et propriété de la société IMAGE, http://www.image-zafar.com/index alceste.htm

7 R project : Logiciel libre pour le calcul statistique, http://www.r-project.org/ 


\section{Résultats et analyses}

La présentation des résultats se fait en deux sous-sections : dans la première, nous présentons l'analyse des discours des apprenants; dans la deuxième, celle des pratiques ou activités réalisées ainsi que celle des productions ainsi obtenues.

\subsection{Analyse des discours}

Dans cette sous-section, nous présentons successivement l'analyse des discussions synchrones, puis le travail de catégorisation des classes de discours lors des verbalisations ; nous présentons ensuite une analyse qualitative de façon globale, puis selon un découpage en sections.

\subsubsection{Les discussions synchrones}

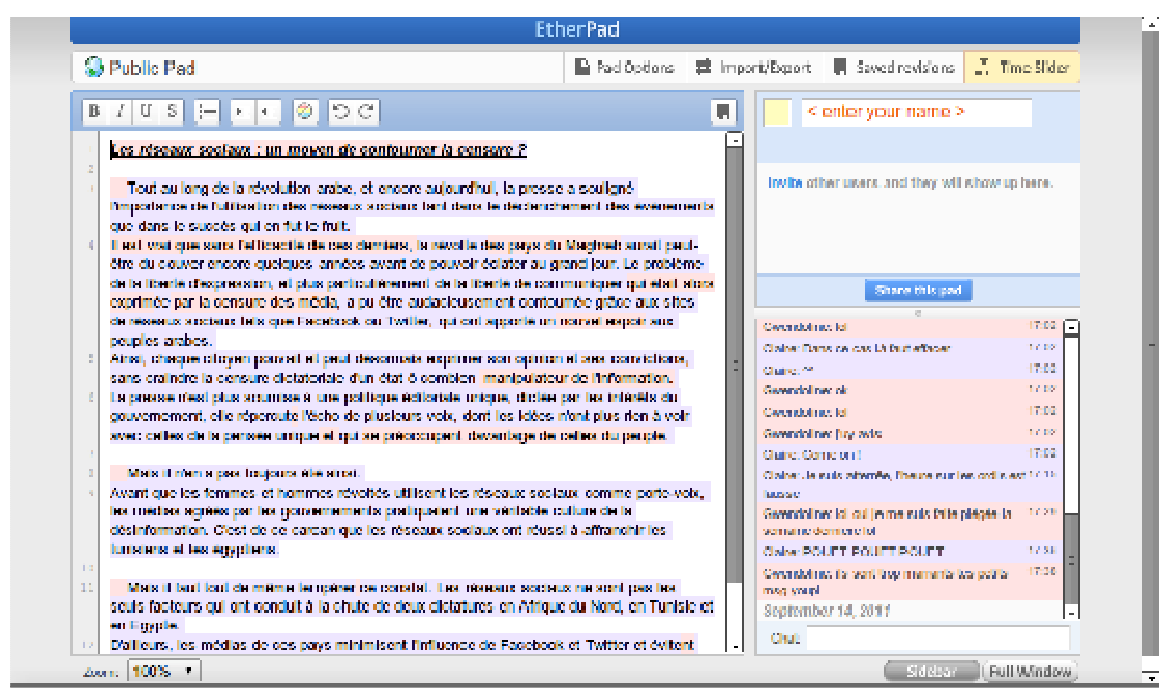

FIG. 1: Le logiciel Etherpad pendant l'expérience

Comme le montre la figure 1, dans Etherpad, le chat est un outil électronique de messagerie instantanée (clavardage) qui apparaît à droite du bloc-note dans lequel les participants rédigent leur texte. Sa fonction première est d'offrir un espace d'échange à propos du texte écrit, favorisant donc la prise de recul et la réflexivité des utilisateurs. Par exemple, sous couvert de convivialité, certaines 
Thibaud HULIN

expressions peuvent donner lieu à un processus de validation du texte en cours (exemple : " je pense qu'il faut mettre que peut et pas pouvait car avant ils pouvaient pas vu qu'il y avait la censure "). Cependant, nous observons que la fonction réflexive du chat, comme outil dédié au travail, n'est pas la seule. D'une part parce que le bloc-note lui-même peut être utilisé comme un outil réflexif de discussion du groupe sur le texte. Ainsi certaines discussions synchrones prennent lieu dans cet espace et non dans l'espace de clavardage, avant d'être effacées cependant. D'autre part parce que les apprenants voient dans la messagerie instantanée un moyen de communication associé à d'autres fonctions : échanges spontanés («Bravo chère collègue »), humour (« Ca c'est une révélation digne de "je suis ton père" »), réflexions plus générales liées au sujet traité ("peut-on dire que les réseaux sociaux ont permis de développer cette révolution? »). En outre, ces formes d'échange permettent aux collaborateurs de maintenir le contact entre eux, conformément à la fonction phatique du langage décrite par Jakobson (1963) (« je lis ce que vous faites »).

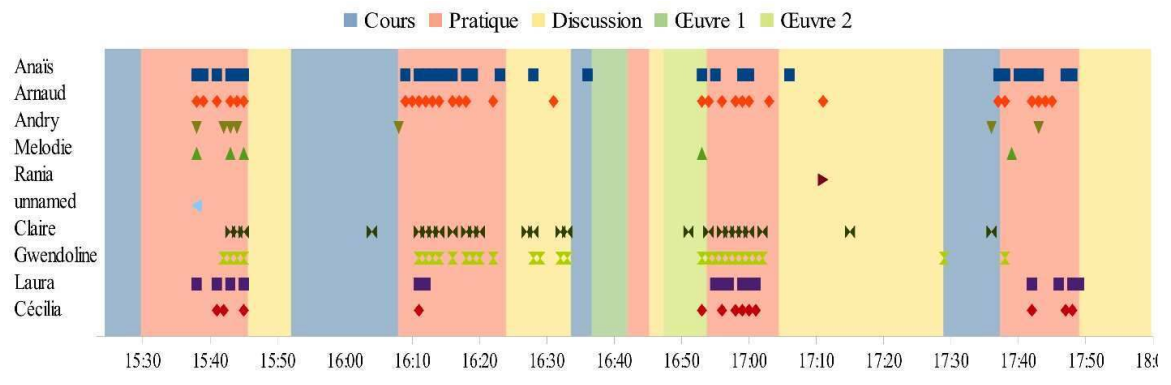

FIG. 2: Vue temporelle sur l'utilisation du chat.

La figure 2 présente une vue temporelle sur chat : les cinq sessions de la formation sont représentées par des couleurs ; les icônes représentent des moments d'échange sur le chat, représentés sur une ligne temporelle pour chacun des participants. Ce schéma montre que si l'utilisation du chat est bien encadrée dans le premier moment de la pratique, elle devient plus fréquente par la suite, en dehors des temps de rédaction du texte principal : il permet donc de maintenir un contact discret entre les collaborateurs. Le chat a-t-il opéré comme un distracteur ? À l'écoute des discussions, les étudiants sont restés concentrés sur l'exercice tout au long de la séance. Le potentiel réflexif du chat est donc important, tout en admettant des usages multiples, qui ne le réduisent pas à un pur outil de diversion. 
Les messages envoyés sur le chat requièrent la plupart du temps une réponse. Un seul apprenant n'utilise pas le chat, ce qui par ailleurs semble avoir pour conséquence de réduire les échanges dans son groupe. Les individus d'un même groupe font en efffet un usage relativement homogène du chat, tandis que les pratiques des groupes diffèrent entre elles.

Certains messages font référence au texte à produire du point de vue "métalinguistique ». Ils concernent la mise en forme du texte du bloc-notes (exemple : «...dans ce cas-là faut effacer »), la méthode employée par les participants («il faut mettre juste les infos qu'on a ») ou les contenus du texte (« peut-on dire que les réseaux sociaux ont permis de développer cette révolution? »). Parmi les messages relevant d'une posture réflexive des apprenants, nous distinguons les messages suivants :

- Des demandes d'avis qui peuvent connoter une demande d'encouragement, exprimer des doutes sur soi ("je pense qu'il ne faut pas mettre censure mais juste culture de la désinformation? ») ;

- Des critiques qui portent sur la syntaxe (« t'es trop fâché avec les points ") ou le style (« on peut pas vraiment dire que ça a déclenché »), lesquelles peuvent alors engendrer de courts débats sur le fond ;

- Des messages qui évoquent l'organisation et qui portent indirectement sur le texte. Dans certains cas, ces messages font apparaître des conflits d'écriture (" on va pas faire l'intro à 2 en $\mathrm{mm}$ temps ") ou des désaccords sur les rôles de chacun (" c'est pas nécessaire de coordonner ») ;

- Dans certains cas, les messages citent le texte (" tu mets "un homme s'est immolé par le feu" ») ou signalent une faute d'orthographe («le mur, les murs ");

- Des messages qui décrivent le niveau de participation de chacun illustré par une couleur (" trop de rose et de violet à ma gauche ça craint »);

- Des messages qui portent sur la structure du texte (« on fait une mini "conclusion" à l'introduction ? ») ou leur longueur (« c'est tout ça l'introduction?");

- Des messages qui portent sur l'expression des idées (" l'idée est là mais pas la formulation ») ;

- Des messages auto-référentiels pouvant favoriser la qualité du travail produit (G: « je te concocte une phrase de ouf lol », C : « c'est quand même un début de phrase super prometteur ") ou qui font référence à 
Thibaud HULIN

la position d'observateur des participants (« je t'observe », « je lis ce que vous faites ").

Dans bien des cas, les messages associent plusieurs fonctions, notamment les fonctions phatiques et expressives signalées par Jakobson (1963). L'idée d'une fonction méta-linguistique nous paraît par ailleurs réductrice dans la mesure où les messages portant sur l'organisation du travail d'écriture ne portent pas à proprement parler sur la langue (niveau méta-linguistique) mais sur ses conditions de production (niveau réflexif).

\subsubsection{Les classes de discours}

Les statistiques lexicales proposées par le logiciel Iramuteq permet d'isoler les trois classes de discours suivantes. La figure (3), produite par le logiciel Iramuteq, montre les relations entre les classes de mots (par couleur) et affiche les mots les plus représentés dans ces classes. La répartition en trois groupes nous permet de les interpréter de la manière suivante. 


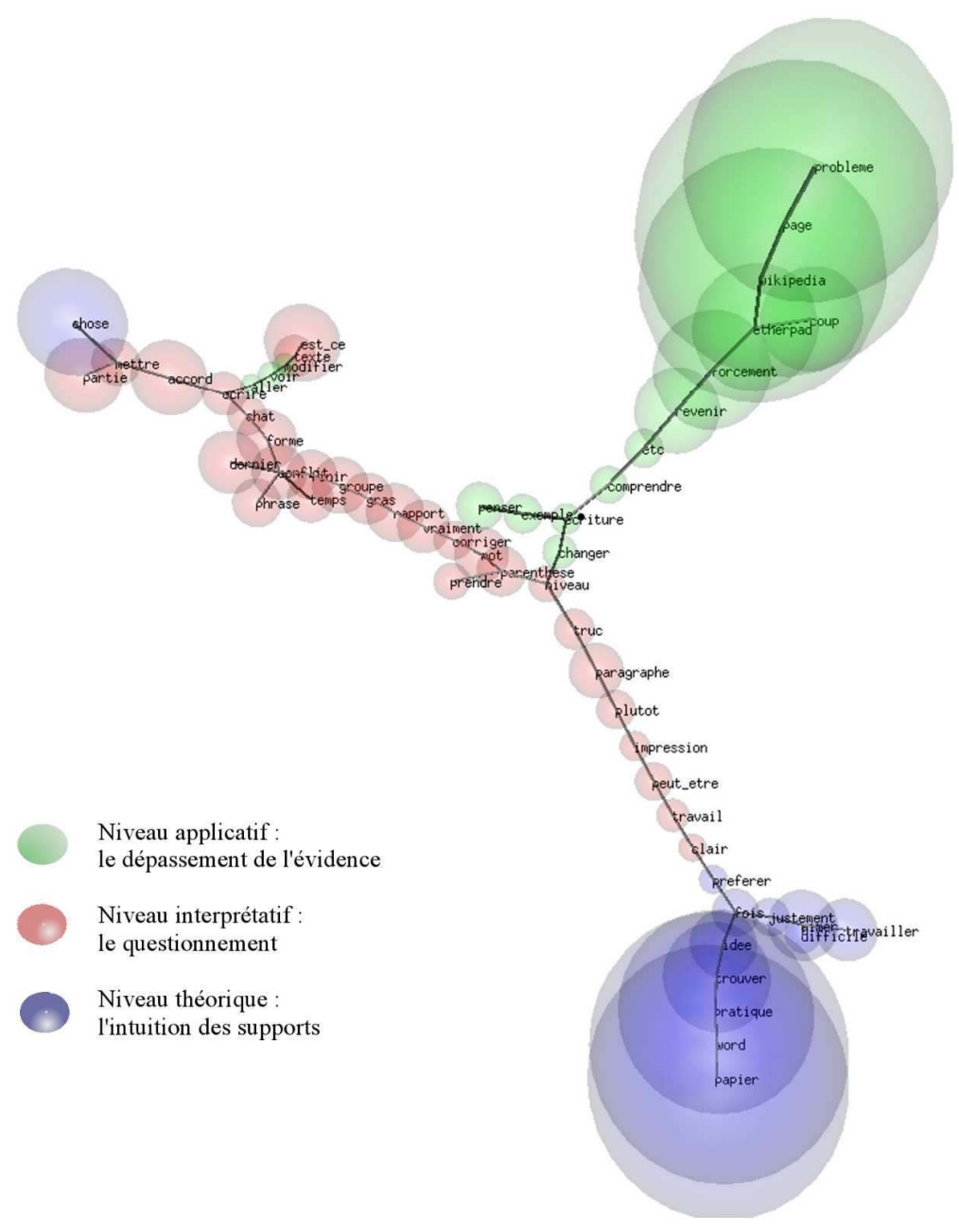

FIG. 3: Graphe 3D des trois « mondes lexicaux » conversationnels

- 1. La classe de discours dominante montre le positionnement des participants, qui privilégient le questionnement (« est-ce...»), mais aussi la recherche d'un " accord ». Les participants sont préoccupés 
Thibaud HULIN

par le « temps ", par exemple, il est fait référence à ce qui est fait “ en dernier " par les différents " groupes ». Ceux-ci évitent de « perdre du temps », d'autant qu'il s'agit de « finir » le travail demandé dans les délais impartis. Les sujets focalisent sur des mises en « rapports ", montrant qu'ils ont intégré l'approche pédagogique comparative. Enfin, l'analyse des « textes ", des «phrases » et des « paragraphes " montre leur attention tournée vers l'écriture et ses mises en " forme » (usage du gras...), ou la prise en compte de légers " conflits " entre auteurs. Les questions de mise en forme et d'ordonnancement correspondent bien au niveau interprétatif de la séance, car c'est ici que la question de l'attention au lecteur est centrale.

- 2. Une seconde classe de discours apparaît à partir de l'étude comparative, réalisée pendant le cours, entre l'encyclopédie Wikipédia et le logiciel collaboratif Etherpad. Cette classe correspond au niveau applicatif. Elle émerge à partir d'un vocabulaire du dépassement de l'évidence ("pas forcément ", " comprendre ") qui entraîne des changements de conceptions à partir d'événements ou d'idées clés (« du coup... »), ce qui implique de parfois « revenir » sur l'activité réalisée.

- 3. Enfin, une troisième classe lexicale est en lien fort avec le niveau théorique, c'est la comparaison entre l'écriture sur « papier » et l'écriture numérique (ce dernier étant assimilé à "Word »). Le lexique soulève la question des supports, par exemple pour savoir s'il est plus « pratique » de « travailler » avec le papier ou le numérique. L'argumentation est peu étayée, et correspond plutôt à un retour d'expérience (on " trouve ", on a " aimé ", etc.). Compte tenu de l'objet traité, cette approche est aussi la plus abstraite des trois : on fait des " choses ", on a des « idées". Contrairement à la partie interprétative, plus critique, la réflexion sur le numérique repose surtout sur l'intuition et le vécu des participants.

Comme le montre la figure (3), la première classe, c'est-à-dire le lexique du questionnement, qui apparaît surtout au niveau interprétatif, domine et entretient davantage de relations avec les deux autres classes. Le questionnement sémiotique domine l'ensemble de la séance.

Au final, l'adéquation des classes de discours avec les trois niveaux de réflexivité abordés montrent l'influence du cours sur la manière dont sont structurés les lexiques des participants. 
Enseigner l'activité « écriture collaborative »

\subsubsection{Les discussions à l'oral}

L'analyse des discussions consiste à observer la place des échanges verbaux dans l'ensemble de la séance, puis à décrire et à comprendre chacun des temps de discussion qui ont eu lieu. L'étude de ces moments est alors structurée du point de vue des différents groupes d'étudiants lorsque cela est nécessaire. Les discussions collectives durent au total cinquante minutes sur les trois heures de cours. La discussion collective représente $34 \%$ du temps contre $37 \%$ de pratique et $29 \%$ de cours, ce qui correspond à un équilibre entre cours, pratique et discussion qui a été recherché dans l'ensemble des séances réalisées.

L'analyse des discussions montre que plusieurs étudiants étaient enthousiastes pour suivre les formations proposées («C'était fun ! - rires... Je trouve que c'est vachement pratique, pour faire un dossier en commun quand on est chacun chez soi »). Dans le cadre d'un échange informel en dehors du cours, une étudiante a expliqué cet intérêt par la possibilité d'appliquer directement cet exercice avec une situation professionnelle, ce qui les changeait des « cours traditionnels".

Les observateurs interviennent de manière à relancer les discussions et la réflexion collective (" et Mélodie ? et Andry ? "). Cette dimension participative peut être la source de rares tensions entre enseignants et apprenants. Ces derniers réagissent soit dans le sens de l'enseignant, souvent lorsqu'il s'agit d'un éclaircissement. Ils peuvent exprimer un désaccord, plus ou moins nuancé (« Non. D'une certaine manière oui, vu qu'on s'entre corrige l'un l'autre. Mais après derrière, moi quand je veux changer quelque chose, je lui disais qu'est-ce que tu en penses. On essayait de pas trop s'imposer... "). Ces tensions montrent que les étudiants ont bien intégré qu'ils n'étaient pas censés être toujours d'accord avec l'enseignant, et qu'on attendait d'eux un vrai recul critique et un engagement personnel. En réponse, les enseignants jouent le jeu en écoutant sans interrompre l'étudiant. Ils l'encouragent à développer ses idées et à réfléchir aux conditions d'un accord avec eux (" et vous qu'est-ce que vous faites avec le chat, est-ce que vous pouvez nous le décrire ? C'est vrai que chaque groupe a un peu ses propres stratégies »). En fin de compte, nous considérons que les observateurs gênent peu les étudiants, ils les stimulent plutôt (« le cours est plus attrayant grâce aux observateurs je trouve lol. Tout à fait, je les aime déjà »). Nous constatons que les étudiants les plus actifs se sont montrés assez libres d'exprimer leurs points de vue, y compris des désaccords, du moment qu'ils pensaient être pertinents. L'objectif qui était de nous intéresser à l'activité réelle et non l'activité prescrite semble donc avoir été compris. 
Thibaud HULIN

Dans leurs discours, les participants font preuve de recul et montrent un effort d'analyse ("C'est plus pour mettre à plat toutes les idées et les voir écrites. Ça nous permettrait d'avoir une idée plus globale du sujet, pas quand on en parle, mais par écrit... par rapport au fait de mettre à plat toutes les idées, ça permet de mieux voir le sujet en sa totalité donc je pense... »). La dimension réflexive du chat favorise le processus d'écriture («En discutant par le chat, on a essayé de se mettre d'accord sur les idées qu'on voulait mettre dedans et quand une commençait une phrase et qu'elle ne savait pas vraiment comment la finir, c'est l'autre qui prenait le relais avec ses idées »). Dans la discussion, les étudiants parviennent à analyser leur activité et à développer cette prise de distance avec une certaine technicité (« elle avait écrit un paragraphe, une phrase, chacun faisait un peu de son côté... Et puis on fait tout pour pas que ça se répète donc elle a changé la tournure de la phrase en effaçant la façon dont elle a écrit et elle a changé la façon dont moi j'avais tourné ma phrase pour pas que ça soit... ce n'était pas un changement d'idées "). Certains locuteurs réfléchissent aux mots qu'ils emploient, comme en témoigne l'expression « entre guillemets " fréquemment utilisée par plusieurs participants. Ainsi, la discussion collective prend le relais du chat, et pousse les participants à davantage réfléchir à leurs propres discours, à l'oral ou à l'écrit. La posture réflexive entraîne donc un phénomène de mise en abyme des discours des participants.

\subsubsection{Les discussions orales par session}

Lors de la découverte d'Etherpad, c'est le plaisir de la découverte de l'autre qui ressort (c'est "fun ", c'est " pratique »). Au-delà de ces sentiments exprimés, ceux qui ont une culture de l'informatique plus avancée se posent des questions déjà plus précises, par exemple sur le fonctionnement des sauvegardes.

Au niveau applicatif, les groupes ont construit des stratégies de rédaction différentes, avec ou sans le chat, mais qui répondent toutes à leur besoin immédiat de coopération.

La discussion issue de l'observation de l'œuvre numérique Venus Poetry porte sur la possibilité de modifier ou non un texte important, comme une poésie ou un article d'encyclopédie. Pour un des apprenants, le poème peut être discuté mais il n'est pas modifiable. En revanche, le savoir appartient à tout le monde et il est donc possible de corriger des textes objectifs. Pour d'autres apprenants, au contraire, la science est affaire de spécialistes, tandis que la poésie est l'affaire de tout le monde («D'une manière on peut considérer que le savoir c'est du domaine du public et le poème appartient à son créateur alors que le savoir appartient à tout le monde... la poésie, en parler, ça me gêne pas, 
Enseigner l'activité « écriture collaborative »

mais la modifier, ça me gêne complètement »). Dans certains cas, l'apprenant n'est pas allé au bout du processus technique, accusant la machine d'avoir enrayer son projet d'édition (« il [l'ordinateur] a pas voulu le mettre »)! Dans ce cas, la discussion a permis de montrer que les machines n'étaient pas le seul obstacle à la collaboration. Leurs représentations peuvent être des obstacles à surmonter, ce qui entraîne des repentirs (" J'admets que comme je n'ai pas I'habitude de changer quoi que ce soit sur Wikipédia du coup j'ai pas bien compris donc »). La discussion permet donc aux étudiants de confronter leurs représentations, et de faire la lumière sur ce qui peut freiner leurs productions.

Au sujet du Mail-roman, des réflexions émergent au sujet du numérique. On compare Etherpad, MS Word et le papier. Les étudiants apprennent à distinguer « ajouter » un contenu et le « modifier ». L'expression " entre guillemets » revient à nouveau dans les discours, ce qui montre le recul que s'efforcent de prendre les participants qui étudient des productions verbales. Grâce à cet effort de distanciation, les apprenants relèvent un certain nombre de changements dans leurs pratiques. Ils se mettent à rédiger ensemble et non pas à côté, organisent la gestion des versions, optimisent l'utilisation du chat ou inversent les rôles scripteur / correcteur (« on a carrément fait une partie en commun ensemble avec chacun... alors que tout à l'heure on a fait une partie chacun et on s'était corrigé : là on a fait vraiment une partie tous les deux. ").

Au niveau interprétatif, qu'il s'agisse d'une simple liste d'idées ou d'un texte structuré, les étudiants améliorent la présentation de leur document, en utilisant les outils de mise en forme (" qu'est-ce que vous avez fait dans cette dernière partie ? - La mise en page »), mais aussi en modifiant leur texte au sujet de la « formulation des phrases »), voire de « reformulations ».

En conclusion de cette partie, l'analyse des classes de discours annonce des différences qualitatives entre les pratiques, entre questionnement permanent, dépassement des évidences et recours à l'expérience et à l'intuition. Les discussions, qui occupent un espace significatif durant le cours, permettent de relativiser les stratégies et les conceptions adoptées initialement dans les groupes. La confrontation des étudiants aux œuvres numériques entraîne a minima des réflexions sur leurs analyses, voire des prises de conscience à propos de leurs freins conceptuels. Dans certains cas, les étudiants peuvent adopter des stratégies nouvelles issues d'un problème lié à la littérature numérique : faut-il juxtaposer les contributions ou écrire un même texte en simultané ? Ainsi, l'effort d'explicitation des étudiants met en évidence l'influence des apports réflexifs sur les pratiques. Ces résultats, déduits de l'analyse des discours, doivent pouvoir se confirmer lors de l'analyse des pratiques et des productions. 
Thibaud HULIN

\subsection{Analyse des pratiques et des productions}

\subsubsection{L'effet d'usage}

Nous avons étudié l'évolution des productions grâce à la fonctionnalité Time Slider d'Etherpad qui permet de rejouer la genèse du texte collaboratif. Nous souhaitons ainsi confirmer l'hypothèse qui est apparue dans l'analyse des discours, à savoir que l'influence du cours implique des changements qualitatifs dans l'ordre des pratiques.

Pour ce faire, nous adopterons un raisonnement par l'absurde et démontrerons la fausseté de l'hypothèse opposée. Selon cette antithèse, les apprenants n'auraient progressé que par eux-mêmes, par simple accumulation d'expérience durant leur pratique et non à partir de l'influence du cours. Nous proposons de mesurer cet " effet d'usage " par la vitesse et le nombre d'interactions de l'apprenant qui, selon l'antithèse, doit augmenter régulièrement, les apprenants devenant de plus en plus à l'aise avec l'outil. $\mathrm{Ce}$ que nous appelons « effet d'usage » n'est pas le résultat de l'expérience réelle, qui est un processus évidemment irrégulier et qui intègre le type d'enseignement reçu. L'effet d'usage est selon nous un phénomène fictif selon lequel utiliser plusieurs fois un logiciel améliorerait nécessairement la productivité, de façon linéaire, et qu'il n'y aurait pas besoin d'un enseignement adapté de type réflexif. Sans doute utiliser de multiples fois un logiciels améliore-t-il la productivité, nous supposons que si l'effet d'usage est opérant, le temps qu'il prend est important et peu efficient, comparé à notre formation de trois heures.

Pour invalider cette hypothèse, nous avons mesuré le nombre d'états enregistrés du texte par le logiciel, appelées les «versions». La production d'une nouvelle version est décidée par le logiciel qui repère de nouvelles interactions effectuées en un temps donné, de l'ordre de deux secondes environ. Pour chaque session de la formation et pour chaque groupe, nous avons comparé la durée de l'ensemble des interactions réalisées avec le nombre de versions produites. Pour valider notre hypothèse, nous ferons le raisonnement suivant : si le nombre de versions par minute progresse linéairement d'une session de pratique à l'autre pour tous les groupes, c'est que les rédacteurs bénéficient de leur expérience et interagissent davantage et plus rapidement. Si l'évolution de cet indicateur n'est pas linéaire, c'est que l'effet d'usage ne suffit pas à rendre compte des stratégies des acteurs. 


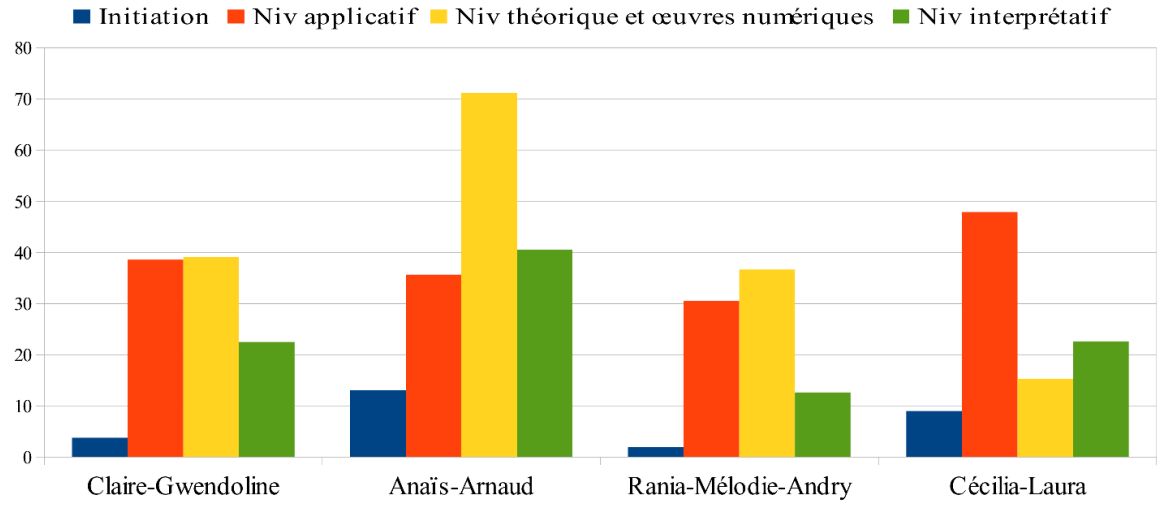

FIG. 4: Productivité des groupes : nombre de versions enregistrées par minute et par session.

La figure 4 montre, pour chaque groupe d'étudiants, le nombre de versions enregistrées durant chacune des sessions de la formation, ces dernières étant représentées par des couleurs. Or, comme le montre le graphique obtenu, ce nombre n'évolue pas linéairement, mais varie en fonction des groupes et des sessions. En particulier, la dernière session de la formation témoigne d'un ralentissement de la production. II n'y a même pas besoin de statistiques pour cela, l' " effet d'usage " est donc un facteur tout à fait relatif pour expliquer les pratiques. On observe plutôt un changement qualitatif des pratiques en fonction de l'apport réflexif fourni en cours, qui vient donc charger l'expérience de l'apprenant.

\subsubsection{La genèse des productions}

Nous étudions maintenant le travail de production des groupes qui a été sectionné en quatre moments : après la phase de découverte, après le niveau applicatif, après le niveau théorique illustré par la littérature numérique, et après le niveau interprétatif.

La première phase est bien sûr un moment de découverte d'un nouveau logiciel, mais aussi de la présence de l'autre à travers la médiation de l'outil numérique. Les participants ne savent pas tout de suite ce qu'ils peuvent modifier, ils ne touchent pas au texte pré-rempli dans un premier temps. L'outil et le texte d'autrui sont "sacrés ", ce qui peut entraîner le silence ou la provocation. 
Thibaud HULIN

Par rapport à la phase précédente, l'étude du niveau applicatif renforce la maîtrise de l'outil et entraîne une augmentation de la productivité de l'apprenant. Cependant, les apprenants travaillent souvent indépendamment les uns des autres, car ils n'osent pas encore se corriger.

La pratique qui fait suite à l'étude du niveau théorique et de la littérature numérique est une phase plus créative et innovante, pas nécessairement plus prolixe que la précédente. Elle montre une prise de recul plus importante de la part de l'apprenant : création de nouveaux paragraphes, nouvelles idées, plus de libertés prises avec le bloc-notes, implication différente des participants.

Au niveau interprétatif, le logiciel continue d'étonner les utilisateurs; par exemple, les outils de mise en forme n'avaient pas forcément été expérimentés au préalable. Tous les groupes mettent en forme leurs textes, même lorsque ces fonctionnalités ont été testées en phase de découverte. Les apprenants interviennent aussi sur la structure du texte, ajoutant des titres au besoin. Cette phase n'est donc pas à proprement parler celle de la " mise en forme " d'un contenu fixé, c'est plutôt le moment d'une relecture attentive qui favorise la compréhension générale du texte pour le lecteur.

L'analyse de la genèse des productions confirme ce qui est apparu dans l'analyse des discussions collectives. Malgré le fait que l'ensemble des temps de pratiques réponde à une même consigne, il existe une différence de nature entre ces moments, plutôt qu'un effet d'usage linéaire. Après une phase de découverte de la médiation d'autrui, tour à tour sacralisée puis reniée, la présentation du niveau applicatif engendre un niveau de maîtrise des fonctionnalités scripturales. Aborder le niveau théorique illustré par la littérature numérique permet alors de renforcer la prise de recul de l'étudiant et ses capacités d'innovation et de créativité. Enfin, la présentation du niveau interprétatif entraîne la relecture du travail de l'apprenant qui prend davantage en compte le lecteur absent, notamment en soignant la présentation de son texte en vue d'une meilleure compréhension par autrui.

\section{Discussion}

Les résultats que nous obtenons montrent que l'enseignement de l'écriture numérique collaborative dans le cadre du dispositif proposé renforce la réflexivité des utilisateurs. En effet, comme le montre l'analyse de l'expérience d'écriture collaborative présentée ici, l'hypothèse d'un « effet d'usage » ne permet pas de rendre compte des changements dans les pratiques des apprenants. On aurait tort, cependant, de nous objecter que l'effet d'usage n'empêche pas des changements de stratégies de la part des apprenants. En effet, nous soutenons qu'il y a bien un effet d'usage, puisque tout usage d'un 
Enseigner l'activité « écriture collaborative »

dispositif entraîne son utilisateur à acquérir une expertise avec le temps. Cependant, nous considérons que l'effet d'usage ne s'oppose pas à la mise en œuvre de stratégies qui sont issues des apports du cours et des niveaux de réflexivité abordés. Une seconde objection pourrait alors consister à montrer que ces ruptures sont dues à des choix personnels de l'apprenant et non pas attribuables au cours. Cependant, l'analyse fine des discours, des pratiques et des productions montrent les liens précis entre les pratiques des sujets et l'enseignement dispensé.

Comme le montre la diversité des usages avec le chat, la communication peut engendrer la distraction, sans être nécessairement un obstacle à l'apprentissage réflexif. Si peu de messages se réfèrent au travail à réaliser, " parler d'autre chose " est aussi un moyen de créer une distance réflexive. On sait que la distraction permet de renforcer la concentration sur le but à atteindre en créant des pauses nécessaires à la qualité du travail. Eigsti et al. (2006) ont montré que les enfants qui savaient se distraire évitaient de céder au désir de consommer trop rapidement une friandise dans le but de doubler la mise. À partir d'un dispositif, nous maintenons que la distraction est susceptible de renforcer le contrôle cognitif et de permettre la connaissance et la maîtrise de soi. Dans les autres observations que nous avons réalisées au collège, nous n'avons pas rencontré d'éléments qui contrediraient fondamentalement cette analyse.

Afin de saisir le processus de construction des connaissances, nous avons construit une expérimentation de type ethno-méthodologique, centrée sur l'analyse fine de l'activité, ce qui ne nous permet pas d'observer des phénomènes réflexifs à plus grande échelle. Dans de futures recherches, nous envisageons d'observer des prises de conscience collectives, selon lesquelles un groupe, qui partage un ensemble de valeurs culturelles, développe la connaissance qu'il a de lui-même. Les phénomènes de réflexivité collective ont été pointé notamment par Bourdieu (1976), qui s'est intéressé à la réflexivité d'un point de vue épistémologique, et repérait une "orchestration des habitus ", le développement de "dispositions durables et transformables » appliqué à l'échelle d'un collectif. La culture politique de certains groupes dans les réseaux sociaux nous paraît fournir un terrain d'étude susceptible de renouveler ce qui n'avait été qu'entrevu par cet auteur. En outre, nous estimons avoir besoin de continuer à resserrer la qualité de notre outillage d'analyse scientifique, notamment sur le plan quantitatif, afin de mieux décrire encore les phénomènes de réflexivité.

Pour terminer, notons que notre dispositif « en présentiel » a été traduit en un dispositif de formation à distance, destiné aux étudiants de l'Université de 
Thibaud HULIN

Paris VIII mais accessible à tous ${ }^{8}$. Trois formations au «Web collaboratif » sont présentées :

- 1. traces et réseaux sociaux : identité numérique et communautés

- 2. publier sur le Web : faire le blog d'un projet ;

- 3. le travail collaboratif : écrire à plusieurs en temps réel (cf. figure $5)$.

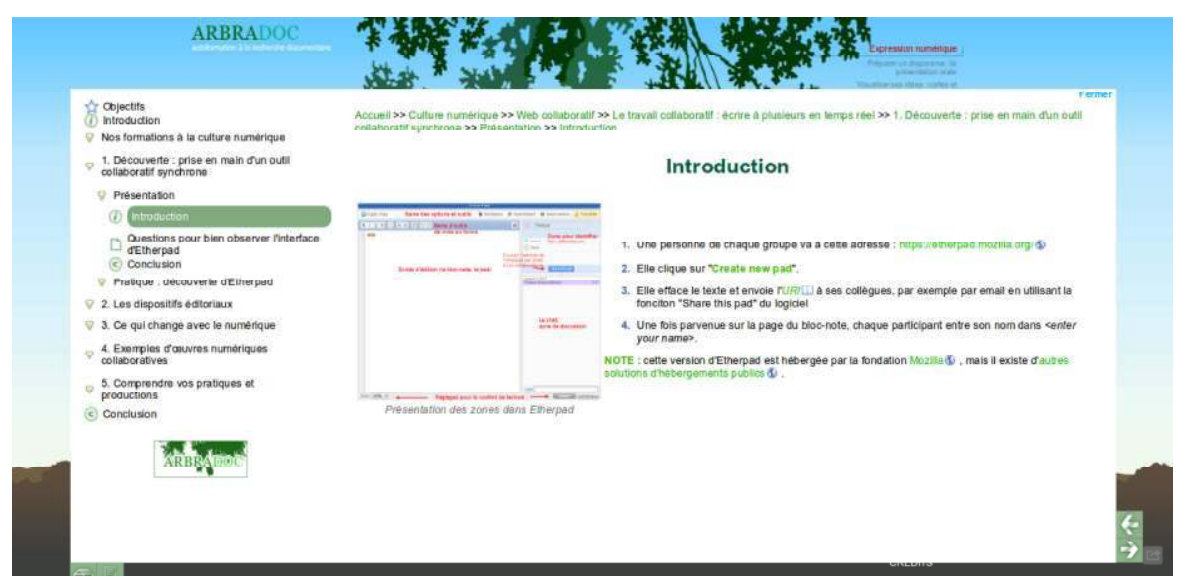

FIG. 5 : Version en ligne de nos formations au « Web collaboratif »

\section{Conclusion}

Afin de répondre à l'injonction publique d'une formation à la collaboration numérique, dont l'écriture est la pierre de touche, nous avons conçu un dispositif de formation à l'écriture collaborative afin de développer les capacités d'analyse fonctionnelle (étude des interfaces), réflexive (analyse de l'activité productive de l'apprenant) et critique ou comparative (analyse des supports, logiciels, processus et productions). L'exemple de l'analyse de la formation " écriture numérique synchrone » permet de valider la pertinence de ce dispositif en montrant que le simple effet d'usage ne suffit pas à expliquer les pratiques des

8 http://arbradoc.bu.univ-paris8.fr 
apprenants, qui développent bien une démarche d'autonomie, sans dépendre de telle ou telle version de logiciel selon un enseignement de type procédural.

Le savoir de l'enseignant n'est ici que le point de départ de l'apprenant. Former à communiquer de façon collaborative via des outils numériques est donc essentiel si l'on veut éviter que l'utilisateur ne dépende de l'état d'avancement d'un logiciel. Savoir analyser les relations de savoir et de pouvoir de tout dispositif numérique collaboratif garantit, selon nous, qu'il développera un véritable savoir-faire technologique.

\section{Références bibliographiques}

ANDRIESSEN, J., BAKER, M. ET VAN DER PUIL, C., 2011, Socio-cognitive tension in collaborative working relations, dans LUDVIGSEN S., LUND A., RASMUSSEN I. et SALJO R. (dir), Learning across sites : new tools, infrastructures and practices, pages 222-242. Routledge, London.

ARNAUD, M. et MERZEAU, L., 2009, Traçabilité et réseaux, vol. 53, Paris, Hermès.

BACHIMONT, B., 2007, Ingénierie des connaissances et des contenus : Le numérique entre ontologies et documents, Paris, Hermès.

BOOTZ, P., 2006, Qu'est-ce que la littérature numérique? $<$ http ://www.olats.org/livresetudes/basiques/litteraturenumerique/1_basiques LN.php>, dernière consultation le 7/10/2012.

BOUCHARDON, S., HULIN, T., CAILLEAU, I., CROZAT, S. et BACHIMONT, B., 2011, Explorer les possibles de l'écriture multimédia, Les enjeux de l'information et de la communication, <http://w3.ugrenoble3.fr/les_enjeux/2011-dossier/Bouchardon-Cailleau-CrozatBachimont-Hulin/index.html>

BOURDIEU, P., 1976, «Le sens pratique », Actes de la recherche en sciences sociales, vol. 2, n91, pp. 43-86.

CHRISTIN, A.-M., 2011, Histoire de l'écriture : de l'idéogramme au multimédia, Paris, Flammarion.

CLOT Y., FAÏTA D., FERNANDEZ G. et SCHELLER L., 2001, « Entretiens en autoconfrontation croisée : une méthode en clinique de l'activité ", Éducation permanente, n946, pp. 17-25. 
Thibaud HULIN

DEROBERTMASURE A. et DEHON A., 2009, « Vers quelle évaluation de la réflexivité en contexte de formation initiale des enseignants ? ", Questions Vives, vol. 6, n92, pp. 29-44.

EIGSTI I., ZAYAS V., MISCHEL W., SHODA Y., AYDUK O., DADLANI M. B., DAVIDSON M. C., ABER J. L. et CASEY B. J., 2006, « Predictive cognitive control from preschool to late adolescence and young adulthood », Psychological Science, vol. 17, pp. 478-484.

ENGESTRÖM Y. 1995, "Objects, contradictions and collaboration, medical cognition : an activity-theoretical perspective ", Artificial Intelligence in Medicine, vol. 7, n5, pp. 395-412.

FLAVELL J. H, 1976, « Metacognitive aspects of problem solving », The nature of intelligence, vol. 12, pp. 231-235.

FOUCAULT M., 1994, Dits et Ecrits, tomes I à IV, Paris, Gallimard."

FOURGOUS J.-M., 2012, « Apprendre autrement » à l'ère numérique. Se former, collaborer, innover : un nouveau modèle éducatif pour une égalité des chances, Rapport de mission parlementaire sur l'innovation des pratiques pédagogiques par le numérique et la formation des enseignants, Paris, Mission Fourgous.

GOODY J., 1979, La raison graphique, Paris, Les Éditions de Minuit.

GUNAWARDENA C. N., HERMANS M. B., SANCHEZ D., RICHMOND C., BOHLE, M. et TUTTLE R, 2009, "A theoretical framework for building online communities of practice with social networking tools », Educational Media International, vol. 46, n9, pp. 3-16.

JAKOBSON R., 1963, "Linguistique et poétique », Essais de linguistique générale, vol. 1, pp. 209-248.

JOINER R. et JONES S., 2003, " Co-ordination and collaboration strategies employed in face-to-face and online discussion ", dans 10th European Conference on Learning and Instruction, 26-30 août, Padoue, Italie.

KRUEGER R. A. et CASEY M. A, 2008, Focus Groups : A Practical Guide for Applied Research, Los Angeles, SAGE Publications Inc.

LLOYD A., 2003, « Information literacy : the meta-competency of the knowledge economy? An exploratory paper », Journal of Librarianship and Information Science, vol. 35, n2, pp. 87-92. 
LOWRY P. B., CURTIS A. et LOWRY M. R, 2004, « Building a taxonomy and nomenclature of collaborative writing to improve interdisciplinary research and practice ", Journal of Business Communication, vol. 41, no1, pp. 66-99.

MANOVICH L., 2002, The language of new media, Cambridge, The MIT press.

OREILLY T., 2007, «What is web 2.0 : Design patterns and business models for the next generation of software ", Communications \& Strategies, n9, pp. 1737.

PERRENOUD P., 2001, Développer la pratique réflexive dans le métier d'enseignant, Paris, ESF.

RATINAUD P. et DEJEAN S., 2009, «IRaMuTeQ : implémentation de la méthode ALCESTE d'analyse de texte dans un logiciel libre », dans Modélisation Appliquée aux Sciences Humaines et Sociales MASHS 2009, 8-9 juin, Toulouse Le Mirail.

SCHÖN D. A, 1994, Le praticien réflexif : à la recherche du savoir caché dans l'agir professionnel, Montréal, éditions Logiques.

SIMONDON G., 1969, Du mode d'existence des objets techniques, Paris, Aubier-Montaigne.

THEUREAU J., 2010, « Les entretiens d'autoconfrontation et de remise en situation par les traces matérielles et le programme de recherche "cours d'action" ", Revue d'anthropologie des connaissances, vol. 4, n2, pp. 287322.

UNION EUROPÉENNE, 2006, Compétences clés pour l'éducation et la formation tout au long de la vie, recommandation 2006/962/CE du Parlement européen et du Conseil, du 18 décembre 2006, sur les compétences clés pour l'éducation et la formation tout au long de la vie, Journal officiel, L 394 du 30/12/2006.

VYGOTSKI L. S, 1985, Pensée et langage, Paris, éditions sociales.

ZHANG L., 2010, What's on your mind?, $<$ https ://www.facebook.com/notes/facebook-data-team/whats-on-yourmind/477517358858>, dernière consultation le 7/10/2012. 